\title{
Mitochondrial reactive oxygen species perturb AKT/cyclin D1 cell cycle signaling via oxidative inactivation of PP2A in low- dose irradiated human fibroblasts
}

\author{
Tsutomu Shimura ${ }^{1}$, Megumi Sasatani², Kenji Kamiya², Hidehiko Kawai², Yohei \\ Inaba $^{1}$, Naoki Kunugita ${ }^{1}$ \\ ${ }^{1}$ Department of Environmental Health, National Institute of Public Health, Saitama, Japan \\ ${ }^{2}$ Department of Experimental Oncology, Research Institute for Radiation Biology and Medicine (RIRBM), Hiroshima University, \\ Hiroshima, Japan \\ ${ }^{3}$ Department of Molecular Radiobiology, Research Center for Radiation Genome Medicine, Research Institute for Radiation \\ Biology and Medicine (RIRBM), Hiroshima University, Hiroshima, Japan \\ Correspondence to: Tsutomu Shimura, e-mail: tsimura@niph.go.jp \\ Keywords: mitochondria, ROS, cyclin D 1, low-dose radiation, AKT
}

Received: August 20, $2015 \quad$ Accepted: November 21, 2015

Published: December 09, 2015

\section{ABSTRACT}

Here we investigated the cellular response of normal human fibroblasts to repeated exposure to low-dose radiation. In contrast to acute single radiation, lowdose fractionated radiation (FR) with $0.01 \mathrm{~Gy} /$ fraction or $0.05 \mathrm{~Gy} /$ fraction for 31 days increased in mitochondrial mass, decreased cellular levels of the antioxidant glutathione and caused persistent accumulation of mitochondrial reactive oxygen species (ROS). Excess ROS promoted oxidative inactivation of protein phosphatase PP2A which in turn led to disruption of normal negative feed-back control of AKT/ cyclin D1 signaling in cells treated with long-term FR. The resulting abnormal nuclear accumulation of cyclin D1 causes growth retardation, cellular senescence and genome instability in low-dose irradiated cells. Thus, loss of redox control and subsequently elevated levels of ROS perturb signal transduction as a result of oxidative stress. Our study highlights a specific role of mitochondrial ROS in perturbation of AKT/cyclin D1 cell cycle signaling after low-dose long-term $\mathbf{F R}$. The antioxidants $\mathrm{N}$-acetyl-L-cysteine, TEMPO and mitochondrial-targeted antioxidant Mito-TEMPO provided protection against the harmful cell cycle perturbations induced by low-dose long-term FR.

\section{INTRODUCTION}

Exposure to ionizing radiation (IR) induces reactive oxygen species (ROS) which affect intracellular metabolic redox control and perturb redox sensitive signaling pathways. Cysteine residues in proteins are a major ROS receptor and sense redox signals to generate sulfenic acid (R-SOH) or cysteine disulfide bonds (-S-S-) from cysteine thiols (R-SH) [1]. Such redox changes on specific proteins can result in altered conformation and/or activity, affecting signaling cascades and cell proliferation.

Mitochondria regulate ATP supply through oxidative phosphorylation. In addition to their pivotal role in cellular metabolism, mitochondria also play essential roles in stress responses and other physiological processes; for instance, mitochondria release cytochrome $\mathrm{C}$ to induce cell death $[2,3]$. As part of their role in the regulation of signal transduction, mitochondria release ROS as second messengers for signaling pathways [4]. A certain amount of ROS is necessary for physiological processes such as differentiation, autophagy and adaptation to hypoxia [3, 5]. In contrast, higher ROS levels inflict oxidative damage to cellular components such as nucleic acids, proteins, and lipids, and inhibit cell proliferation. Mitochondrial ROS-mediated oxidative stresses are associated with induction of genomic instability in irradiated cells $[6,7]$. However, the precise mechanisms underlying radiationinduced genome instability are not fully understood. In order to control ROS levels, mitochondria harbor antioxidant defense systems such as glutathione (GSH) and manganese superoxide dismutase (MnSOD) which 
scavenge ROS $[8,9]$. Dismutation of superoxide anions in the mitochondria forms $\mathrm{H}_{2} \mathrm{O}_{2}$ either spontaneously or through the catalytic function of MnSOD in order to maintain the intracellular redox environment. Glutathione peroxidases then further reduce $\mathrm{H}_{2} \mathrm{O}_{2}$ to water by using glutathione as a ROS receptor.

AKT kinases are critical players in PI3K-mediated signal transduction pathways. In response to growth signals, AKT is activated downstream of PI3K and phosphorylates downstream substrates to transmit growth signals [10]. Active AKT mediates cell proliferation, cell survival, and cellular metabolism through multiple intracellular signaling pathways $[11,12]$. DNA damage responses (DDR) have been well investigated using acute single radiation (SR) at high doses [13]. Following SR, AKT was shown to be transiently activated and then converted to an inactive dephosphorylated form 24 hours after irradiation [14]. AKT is dephosphorylated and inactivated by several phosphatases, including protein phosphatases 2A (PP2A), PH domain leucine-rich repeat protein phosphatase (PHLPP) and phosphatase with tensin homology (PTEN) [15-18]. DNA damage signaling generally stops when damaged DNA is repaired. In contrast to SR, DNA damage may accumulate with increasing cumulative radiation doses by repeated exposure, and the DDR may persist over a prolonged period. However, the cellular responses to long-term low doses of radiation remain uncertain due to lack of sufficient study. Recently, we have found that AKT activation persists over a prolonged period following long-term fractionated radiation (FR) for 31 days [14]. The radioresponse of AKT is changed from transient activation to constitutive activation according to duration of radiation exposure. PP2A, the phosphatase which is a negative regulator of AKT activity, is itself inactivated by oxidation of cysteine residues $[19,20]$. Thus, ROS-mediated oxidative stresses might be involved in perturbations of the AKT signaling pathway via oxidative PP2A inactivation.

In this study, normal human fibroblasts were exposed to low doses $(0.01 \mathrm{~Gy}$ or $0.05 \mathrm{~Gy} /$ fractions) of FR for 31 days. Low-dose long-term FR suppressed cell growth and induced cellular senescence with accumulation of mitochondrial ROS. Coincidently, PP2A activity was down-regulated in long-term FR cells. We found that long-term FR induced oxidative inactivation of PP2A via accumulation of mitochondrial ROS, thus leading to perturbations of the AKT pathway.

\section{RESULTS}

\section{Increase in mitochondrial mass after low-dose long-term FR}

We exposed MRC-5 and TIG-3 normal human fibroblast cells to $0.01-$ or 0.05 -Gy fractions of X-rays. Cells exposed to FR for 31 days were referred to as $31 \mathrm{FR}$ cells. The morphological observations of mitochondria and FACS analysis with MitoTracker Green FM staining revealed that 31FR MRC-5 and 31FR TIG-3 cells exhibited an increase in mitochondrial mass compared to unirradiated 0FR cells (Figure 1A). The expression of PPAR- $\gamma$ co-activator-1 $\alpha$ (PGC1- $\alpha)$ which stimulates mitochondrial biogenesis was examined in 31FR TIG-3 cells by western blotting (Figure 1B). Consistent with the result of increase in mitochondrial mass, the PGC1- $\alpha$ expression elevated after low-dose long-term FR in 31FR cells. Cells were continuously treated with $\mathrm{N}$-acetyl-L-cysteine (NAC) to mitigate oxidative stresses at a final concentration of $1 \mathrm{mM}$ during FR exposure. Medium changes were made at 2- or 3 -days intervals. Continuous NAC treatment prevented increase in mitochondrial mass and induction of PGC1- $\alpha$ expression in 31FR cells (Figure 1A, 1B).

\section{Accumulation of mitochondrial ROS in low-dose FR cells}

ROS are produced in mitochondria as a by-product of ATP production through oxidative phosphorylation. Therefore, we investigated the effect of increase in mitochondrial mass on generation of ROS after long-term FR. We measured the amounts of ROS in cells by staining them with 2',7'-dichlorofluorescin diacetate(DCFD) 24 hours after last FR at indicated days (Figure 2A). Distribution of DCFDA-stained cells was unchanged between unirradiated cells and FR cells until 14 days, while strong DCFDApositive cells appeared when cells were exposed to FR for $>21$ days and were further increased at 31 days (Figure 2A, upper left panel, FR). In contrast, ROS accumulation was not observed in TIG-3 cells exposed to 2 Gy of SR (Figure 2A, upper right panel, SR). ROS were also not induced in TIG3 cells cultured for 31 days without irradiation (Figure 2A, dotted black line, upper left panel, FR). Similarly, induction of ROS was observed after low-dose long-term FR in MRC-5 cells when cells were exposed to FR for $>21$ days but not after SR (Figure S1A). Induction of ROS was not observed by continuous NAC treatment in MRC- 5 and TIG-3 cells regardless of FR exposure (Figure 2A, red line, lower right panel, FR+NAC and Figure S1A). Furthermore, ROS accumulation was suppressed by treatment with other antioxidants 2,2,6,6-Tetramethylpiperidine 1-Oxyl (TEMPO) or (2-(2,2,6,6-Tetramethylpiperidin-1-oxyl-4ylamino)-2-oxoethyl)triphenylphosphonium chloride (MitoTEMPO) for 24 hours in TIG-3 31FR cells (Figure 2B).

Mitochondrial ROS were stained with MitoSOX-red which is a fluorogenic dye for highly selective detection of superoxide in mitochondria. Strong intensity of MitoSOXred staining was evident in 31FR TIG-3 cells treated with $0.01 \mathrm{~Gy} /$ fraction, but not in unirradiated control cells (Figure 2C upper panel). MitoSOX-red positive cells were also evident in 31FR MRC-5 cells (Figure S1B). NAC and Mito-TEMPO treatment eliminated MitoSOX-red staining 
in 31FR cells (Figure 2C middle panel and lower panel). Thus, mitochondrial ROS are generated in 31FR cells with increase in mitochondrial mass (Figure 1).

\section{Oxidative mitochondrial DNA damage and decrease in GSH levels after low-dose long-term FR}

We next quantified oxidative damage on nuclear and mitochondrial DNA after low-dose long-term FR by measuring amounts of apurinic/apyrimidinic (AP) site using Nucleostain DNA damage Quantification Kit. As shown in Figure 3A on left panel, the number of APsites in mitochondrial DNA greatly increased in $31 \mathrm{FR}$ cells compared to unirradiated OFR cells. Thus, oxidative mitochondrial DNA damage persisted at least for 24 hours following low-dose long-term FR. Accumulation of AP sites in nuclear DNA was not observed among all four samples regardless of treatment (Figure 3A, right panel). These results indicated that mitochondrial
DNA is more sensitive to oxidative stresses triggered by low-dose long-term FR than nuclear DNA in human cells. Accumulation of oxidative mitochondrial DNA damage was suppressed by continuous NAC treatment in 31FR MRC5 and 31FR TIG-3 cells (Figure 3A). We further evaluated oxidative DNA damage in nuclear and mitochondrial DNA after low-dose long-term FR by measuring amounts of 8-Hydroxydeoxyguanosine (8-OHdG) using high performance liquid chromatographyelectrochemicaldetector (HPLC-ECD). As shown in Figure 3B, 8-OHdG accumulated in the mitochondrial DNA of TIG-3 31FR cells compared to that in unirradiated TIG-3 OFR cells. In contrast, 8-OHdG levels were low in nuclear DNA of 0FR and 31FR cells of TIG-3 regardless of FR exposure.

We next investigated the effect of long-term FR on antioxidant defense systems against mitochondrial ROS. Amounts of the cellular antioxidant GSH were measured in unirradiated control OFR cells and 31FR cells. GSH levels were unchanged at 24 hours after 2 Gy of SR in MRC-5 and TIG-3 cells (Figure 3C, lower panel). In contrast, GSH levels decreased in MRC-5 31FR cells

A

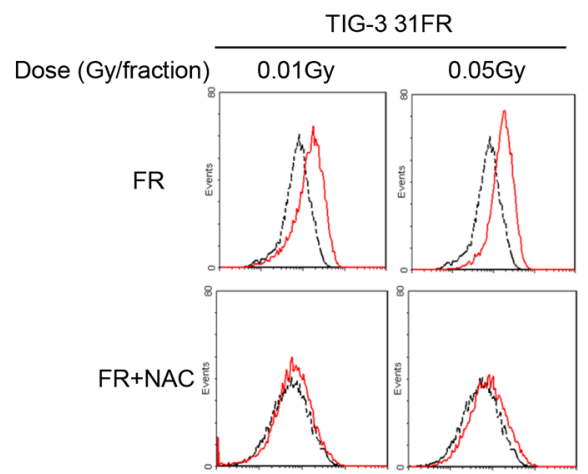

MitoTracker staining

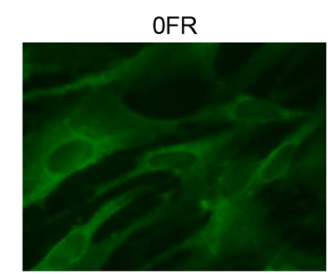

OFR+NAC

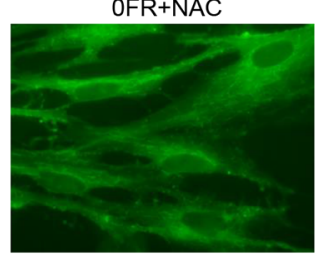

$0.01 \mathrm{~Gy}+\mathrm{NAC}$
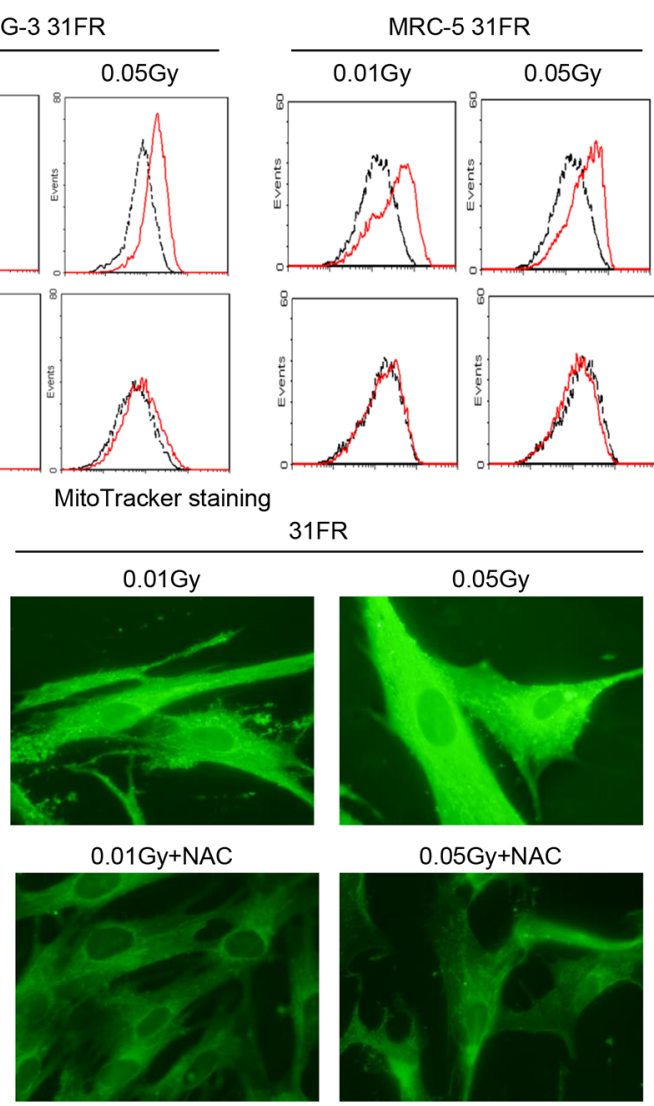

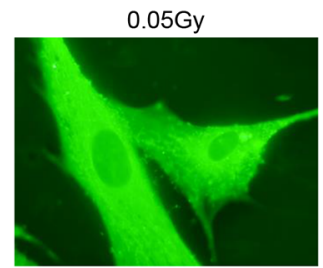

$0.05 \mathrm{~Gy}+\mathrm{NAC}$

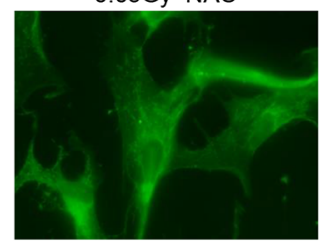

TIG-3 31FR
\begin{tabular}{|c|c|c|}
\hline $\begin{array}{c}\text { Dose } \\
\text { (Gy/fraction) }\end{array}$ & FR & FR+NAC \\
\hline 0 & 9.0 & 8.0 \\
0.01 & 18.2 & 9.1 \\
0.05 & 19.2 & 11.7 \\
\hline
\end{tabular}

MRC-5 31FR
\begin{tabular}{|c|c|c|}
\hline $\begin{array}{c}\text { Dose } \\
\text { (Gy/fraction) }\end{array}$ & FR & FR+NAC \\
\hline 0 & 17.5 & 17.4 \\
0.01 & 47.5 & 16.7 \\
0.05 & 34.8 & 19.0 \\
\hline
\end{tabular}

\section{B}

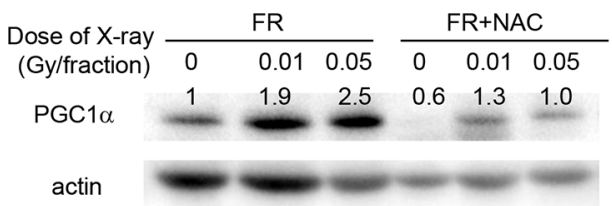

Figure 1. Mitochondrial mass, oxidative DNA damage in mitochondrial DNA and GSH levels in low-dose long-term FR cells A. Images of MitoTracker Grenn FM staining in untreated 0FR and 31FR cells of TIG-3 were shown on the lower panel. On the upper panel, FACS results for MitoTracker Green FM staining in untreated 0FR (dotted black lines) and 31FR cells (red lines). Mean fluorescence intensity values of the MitoTracker staining were shown on the upper right panel. B. Western blotting of PGC1 $\alpha$ and actin was shown in 0FR and 31FR cells with and without NAC. The amounts of PGC1 $\alpha$ were normalized by corresponding actin level. The values are expressed relative to the control value of 0FR cells. 
and TIG-3 31FR cells compared to that of unirradiated control cells (Figure 3C, upper panel). Decrease in GSH levels after low-dose long-term FR was not observed in continuous NAC-treated 31FR cells.

\section{Perturbation of AKT signaling via down- regulation of PP2A activity after long-term FR}

ROS oxidize phosphatases and modulate cellular signaling pathways [21]. The effect of ROS accumulation on AKT signaling was investigated by examination of phosphorylation of AKT on serine 473 in FR-treated MRC-5 cells. Consistent with our report in other cell types [14], AKT in MRC-5 cells was transiently activated by 2 Gy of SR, with levels of active AKT declining to control levels by 24 hours (Figure S2). AKT activation was evident at least 24 hours after the last FR treatment when cells were exposed to 0.01 Gy and 0.05 Gy of FR for 31 days, consistent with the timing of ROS accumulation (Figure 4A). Continuous NAC treatment suppressed AKT activation in MRC-5 31FR cells (Figure 4A). These results

A

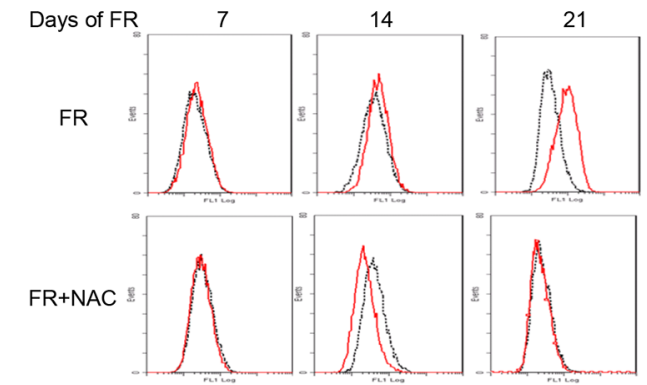

DCFDA staining

\begin{tabular}{|ccccc|}
\multicolumn{1}{l}{ TIG-3 } & \multicolumn{5}{c|}{} \\
\hline Days of FR & 7 & 14 & 21 & 31 \\
\hline OFR & 2.6 & 4.6 & 4.1 & 4.8 \\
O.01FR & 2.8 & 5.7 & 11.0 & 16.4 \\
OFR+NAC & 4.1 & 5.6 & 1.5 & 3.5 \\
0.01FR+NAC & 3.6 & 2.8 & 2.7 & 3.5 \\
\hline
\end{tabular}

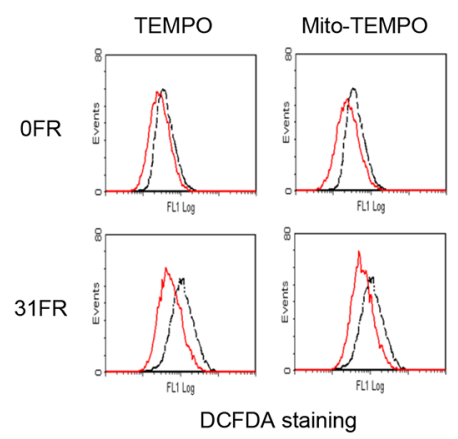

suggest that accumulation of mitochondrial ROS affects AKT activation after long-term FR.

The protein phosphatase PP2A plays a key role in controlling AKT inactivation through its dephosphorylation of AKT on both Serine 473 and Threonine 308 [18]. We measured PP2A activity after low-dose long-term FR. PP2A activity was attenuated in 31FR MRC-5 and 31FR TIG-3 cells compared to that of control cells (Figure 4B). NAC treatment prevented downregulation of PP2A activity in 31FR cells (Figure 4B). PP2A activity was restored by DTT treatment to control levels (Figure 4B).

Protein phosphatases are known to be susceptible to a number of redox-dependent modifications, including an interchange between the reduced thiol and several different oxidized disulfide states. Thus, we hypothesized that in cells subjected to long-term FR, ROS may oxidize PP2A on its active-site cysteine, thus inactivating PP2A. Disulfide bond formation in PP2Ac was examined by diagonal electrophoresis with western blotting (Figure 5). PP2A with an intrarmolecular disulfide (above the diagonal) was detected in $31 \mathrm{FR}$ cells but not in 0FR cells.

C
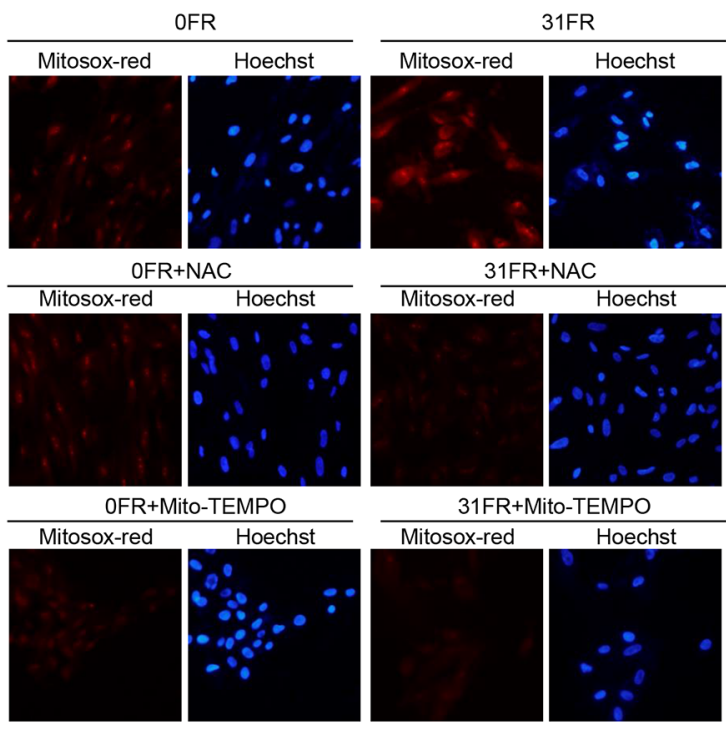

31FR+Mito-TEMPO

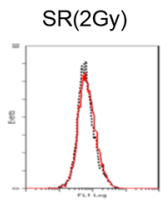

DCFDA staining
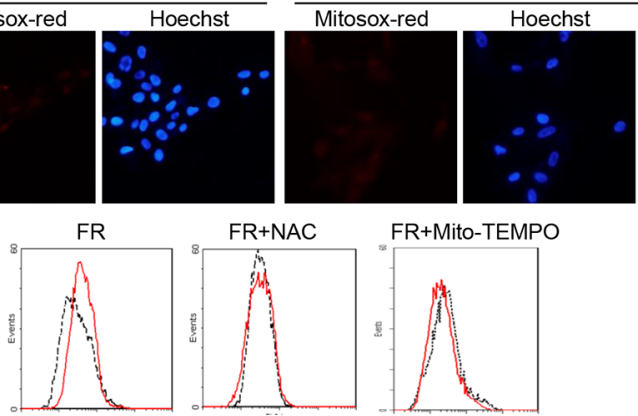

\begin{tabular}{|c|c|c|c|}
\hline Dose (Gy/fraction) & FR & FR+NAC & FR+Mito-TEMPO \\
\hline 0 & 4.5 & 3.8 & 4.6 \\
\hline 0.01 & 5.3 & 3.7 & 3.1 \\
\hline
\end{tabular}

Figure 2. ROS generation in OFR and 31FR cells A. FACS results for DCFDA staining in untreated (dotted black lines) and treated TIG-3 cells (red lines). Mean fluorescence intensity values of DCFDA staining were shown. B. FACS results for DCFDA staining in untreated OFR and 31FR cells (dotted black lines) and TEMPO- or Mito-TEMPO-treated TIG-3 0FR and 31FR cells (red lines). C. Images of Mitosox-red staining cells in control 0FR and 31FR cells of TIG-3 with and without NAC or Mito-TEMPO treatment. Mean fluorescence intensity values of Mitosox-red staining were shown. 
NAC treatment prevented disulfide bond formation of PP2A in 31FR cells (Figure 5).

\section{Abnormal nuclear cyclin D1 accumulation in long-term FR cells}

We have previously reported that perturbation of AKT signaling causes persisted nuclear cyclin D1 expression during DNA replication in normal human fibroblasts [22]. To detect abnormal nuclear accumulation of cyclin D1 in S-phase, cells were treated with a hypotonic buffer containing detergent to remove cytoplasmic cyclin D1. Proliferating cell nuclear antigen (PCNA) immunofluorescence was used to identify cells in S phase [22]. Detergent-insoluble nuclear cyclin D1 was detected in PCNA-positive nuclei of cells exposed to 0.05 Gy of FR for 31 days (Figure $6 \mathrm{~A}$ upper right panel). We have previously reported that in 31FR MRC-5 and TIG-3 cells, the percentage of cyclin D1 and PCNA double positive cells is about 30\% [22]. Thus, perturbation of AKT signaling after longterm FR would be expected to result in abnormalities in cell cycle control. Treatment with antioxidants such as NAC, TEMPO or Mito-TEMPO suppressed induction of abnormal nuclear cyclin D1 in 31FR cells (Figure $6 \mathrm{~A}, 6 \mathrm{~B}$ and $6 \mathrm{C})$.

\section{Growth suppression and induction of cellular senescence after low-dose FR}

We next analyzed the cell growth of FR-treated cells after administration of the antioxidant NAC. Exposure to low-dose FR decelerated cell proliferation in both cell lines as we reported previously (Figure 7A left panel, Figure S3) [22]. NAC was shown to rescue growth retardation induced by low-dose long-term FR (Figure 7A right panel, Figure S3).

Cellular senescence is thought to be induced by ROSmediated oxidative stress after long-term FR. Therefore, we investigated cellular senescence in response to FR. Senescent cells were identified by detection of senescenceassociated $\beta$-galactosidase ( $\beta$-gal) activity. Figure 7B depicts representative $\beta$-gal staining in MRC- 5 cells irradiated with $0.05 \mathrm{~Gy} /$ fraction for 31 days. The percentage of $\beta$-galpositive senescent cells increased remarkably in both MRC5 and TIG-3 cells treated with FR for 31 days compared to cells cultured for 31 days without FR (Figure 7C). PML bodies, a marker for senescent cells were evident in 31FR cells but not in 0FR cells (Figure S4). Thus, growth retardation induced by low-dose long-term FR was partially due to an increase in senescent cells. Treatment with the antioxidant NAC eliminated this FR-induced increase in senescence. (Figure 7B, 7C). Our results indicate that oxidative stress mediates growth retardation and cellular

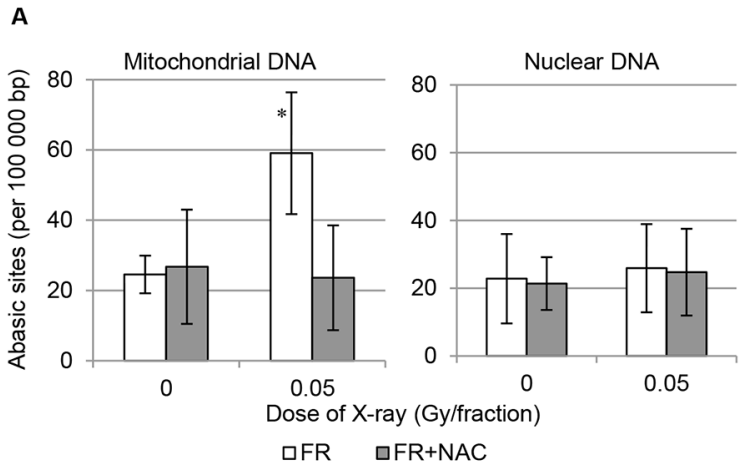

C
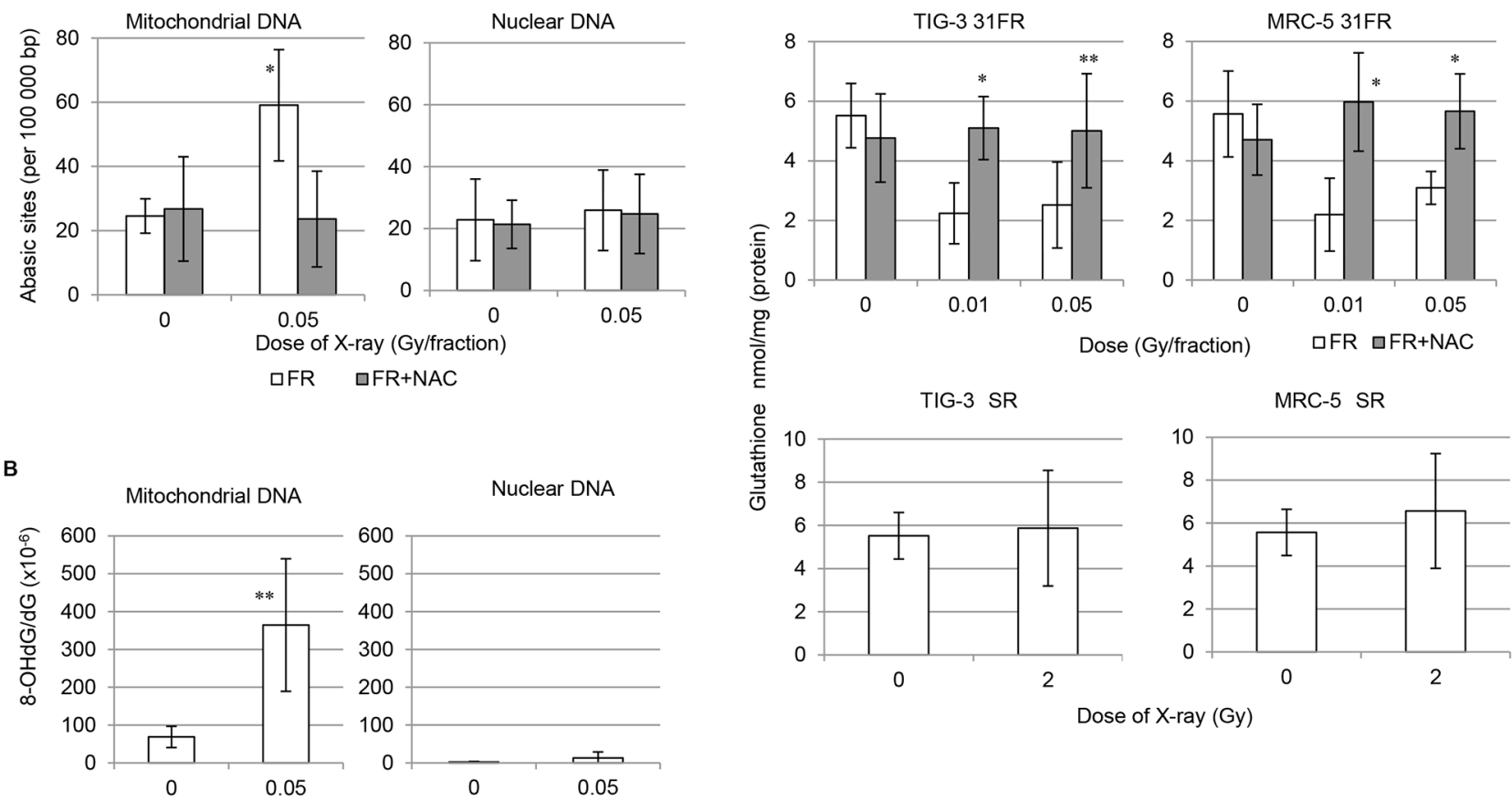

Figure 3. Mitochondrial DNA damage and GSH levels in OFR and 31FR cells A. The number of abasic sites was measured by the DNA damage quantification assay performed on nuclear and mitochondrial DNA in control 0FR and 31FR cells of TIG-3 with and without NAC treatment. B. 8-OHdG levels on nuclear and mitochondrial DNA in control 0FR and 31FR cells of TIG-3. C. Cellular GSH levels were shown for control and 31FR TIG-3 cells in the upper left panel and for control and 31FR-treated MRC-5 cells in the upper right panel. Cellular GSH levels were shown in unirradiated cells and at 24 hours after 2-Gy irradiation for TIG-3 cells in the lower left panel and for MRC-5 in the lower right panel. 
senescence of MRC-5 and TIG-3 cells after low-dose longterm FR.

\section{DISCUSSION}

Here, we demonstrate that low-dose long-term FR causes increase in mitochondrial mass and persistently increased levels of mitochondrial ROS in normal human cells. Mitochondria express the antioxidant GSH to protect cells against oxygen toxicity [9]. Cellular GSH levels are decreased by GSH consumption and lost through efflux in response to oxidative stress and many pathological conditions [23]. We here demonstrated that cellular GSH was decreased by low-dose long-term FR. Insufficient GSH levels lead to a decrease in antioxidant capacity, accumulation of mitochondrial ROS, and stimulation of long-lasting oxidative stresses in longterm FR cells. NAC serves as a precursor of cysteine and augments intracellular levels of GSH [24]. Therefore, oxidative stresses after long-term FR were reversed by administration of NAC. GSH deficiency is associated with increased hemolysis, cataracts and central nervous system abnormalities [9]. Interestingly, GSH depletion and mitochondrial dysfunction are induced in response to very low-dose chronic exposure to persistent organic pollutants such as 2,3,7,8-Tetrachlorodibenzo- $p$-dioxin $[25,26]$.

Here we found that AKT activation was persistent in normal human fibroblasts treated with long-term FR, but not in cells treated with 2 Gy of SR. Coincidently, mitochondrial ROS accumulated in these long-term FR cells. Therefore, persistent oxidative stresses may affect AKT signaling after long-term FR. AKT inactivation is
A

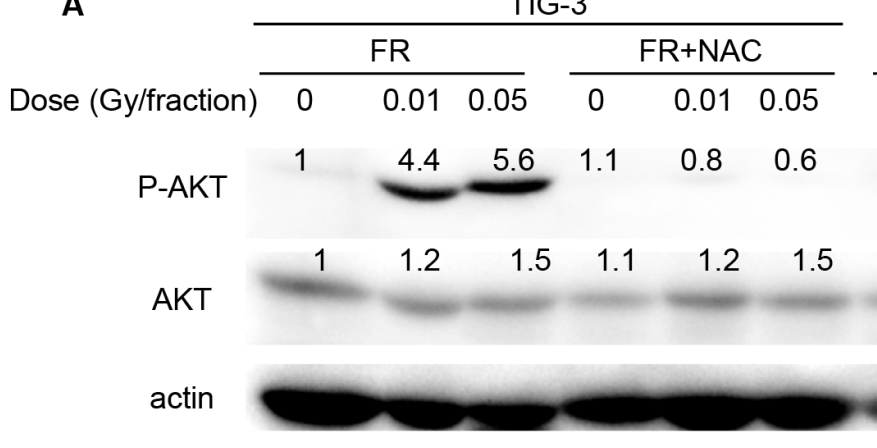

B

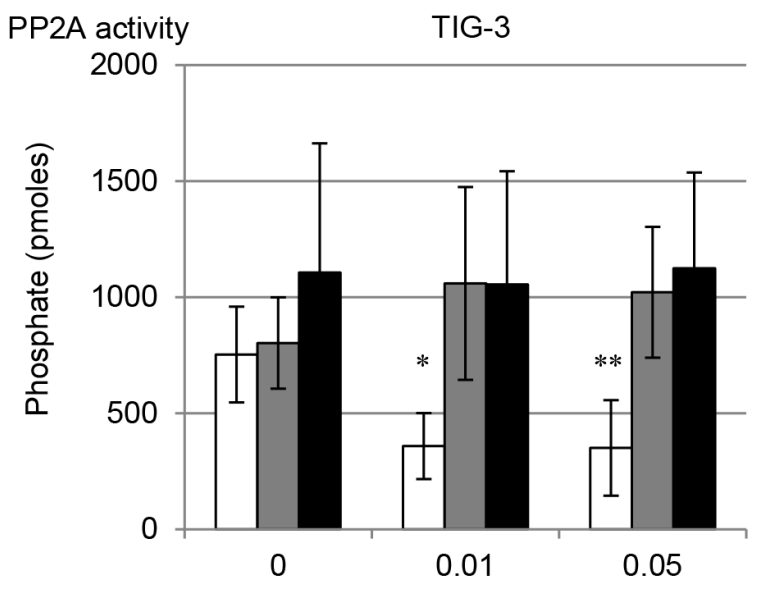

MRC-5

\begin{tabular}{|c|c|c|c|c|c|}
\hline \multicolumn{3}{|c|}{ FR } & \multicolumn{3}{|c|}{$\mathrm{FR}+\mathrm{NAC}$} \\
\hline 0 & 0.01 & 0.05 & 0 & 0.01 & 0.05 \\
\hline 1 & 30 & & 1.3 & 1.6 & 1.5 \\
\hline
\end{tabular}

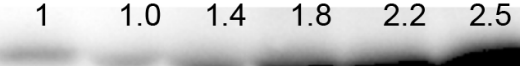
(n)
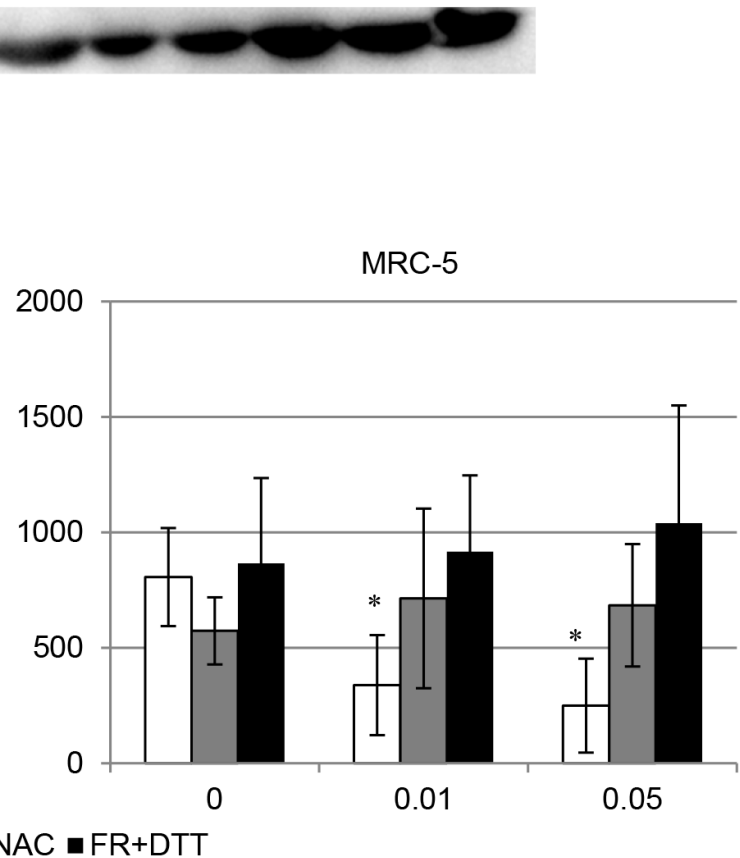

Dose (Gy/fraction)

Figure 4. AKT activation after long-term FR AKT activation via down-regulation of PP2A activity after low-dose longterm FR A. AKT phosphorylation on Ser473 in TIG-3 31FR ells and MRC-5 31FR cells with and without NAC treatment. The amounts of total AKT and p-AKT were normalized by corresponding actin level. The values are expressed relative to the control value of $0 \mathrm{FR}$ cells. B. PP2A activity in unirradiated controls and cells treated with 31 days of FR ( $0.01 \mathrm{~Gy} /$ fraction and $0.05 \mathrm{~Gy} /$ fraction $)$ with and without NAC. For DTT-treated samples, DTT (1 mM) was added to the lysis buffer. 
mediated by dephosphorylation of the protein via several phosphatases including PP2A [18]. However, ROS oxidizes PP2A on cysteine residues and down-regulates PP2A activity in long-term FR cells. This loss of PP2A activity can thus lead to a loss of negative feedback control of the AKT pathway, leading to persistent, longlasting AKT activity in cells after long-term FR.

We here demonstrated that mitochondrial ROS perturbed the AKT/cyclin D1 pathway after longterm FR. Perturbation of AKT signaling and cyclin D1 expression is shown to be implicated in tumoregenesis due to inappropriate cell cycle entry [10, 27, 28]. Aberrant cyclin D1 expression provides a driving force behind the development of tumorigenesis and is often detected in premalignant and malignant tissues. Abnormal nuclear cyclin D1 accumulation in S phase induces DNA replication stresses and resulting DNA double strand breaks, and is associated with induction of genomic instability in irradiated cells [22, 29]. Perturbation of cyclin D1 expression also associates with cellular senescence [30, 31]. Data from this current study indicates that induction of nuclear cyclin D1 accumulation was suppressed by treatment with antioxidants NAC, TEMPO and Mito-TEMPO in 31FR MRC-5 and TIG-3 cells. Thus, perturbation of the AKT/cyclin D1 pathway after low-dose long-term FR is attributable to ROS-mediated oxidative stress. Increasing antioxidant capacity is critical to preventing abnormalities in cyclin D1 expression after long-term FR.

In conclusion, we have demonstrated a link between mitochondrial dysfunction and perturbation of the AKT/cyclin D1 cell cycle signaling in low-dose irradiated human cells. Mitochondria are the target organelle for low-dose radiation. Administration of antioxidants may be effective in mitigating the toxicity of low-dose long-term FR in order to guard genome stability in irradiated cells. Our findings will provide new insights in cancer risk estimation associated with long-term low-dose radiation exposure.

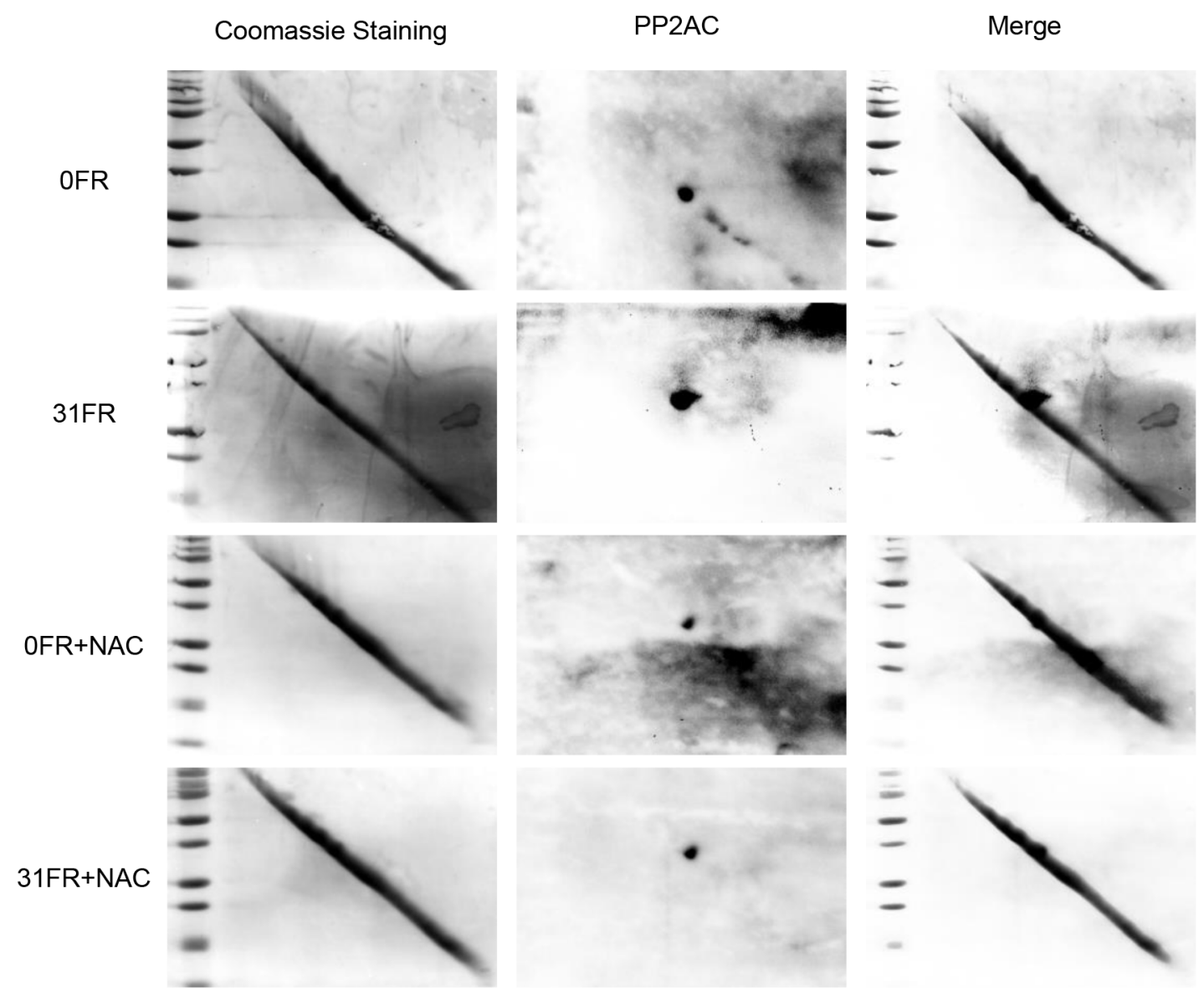

Figure 5 Disulfide modification of PP2A Cell lysates were collected from TIG-3 cells at 24 hours after the last FR treatment Proteins separated by diagonal electrophoresis were stained with Coomassie blue (left panel). Western blotting of PP2AC was shown on the right panel in OFR and 31FR cells with and without NAC. 


\section{METHODS}

\section{Cell culture conditions and drugs}

Normal human diploid lung fibroblasts (MRC5 and TIG-3) were purchased from the Health Science Research Resources Bank (Osaka, Japan), and grown in minimum essential medium (Nacalai Tesque, Kyoto, Japan) supplemented with $10 \%$ heat-inactivated fetal calf serum. Serial passage experiments were started after 33 or 23 population doublings for MRC- 5 and TIG-3 cells, respectively. NAC and Mito-TEMPO were purchased from Sigma (San Diego, CA). TEMPO was purchased from Tokyo Chemical Industry Co., Ltd (Tokyo, Japan). Cells were treated with TEMPO or Mito-TEMPO at a final concentration of $100 \mu \mathrm{M}$ for 24 hours.
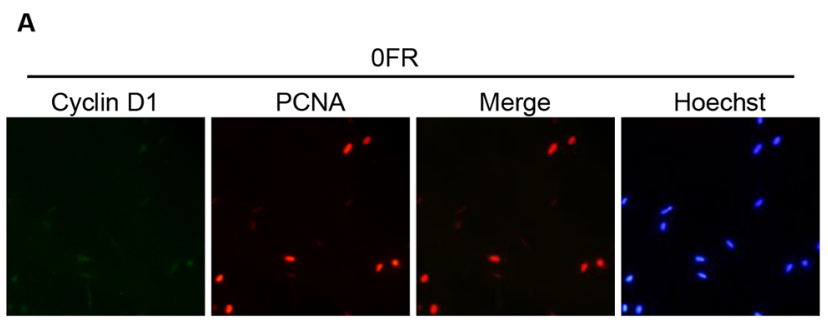

$\mathrm{OFR}+\mathrm{NAC}$

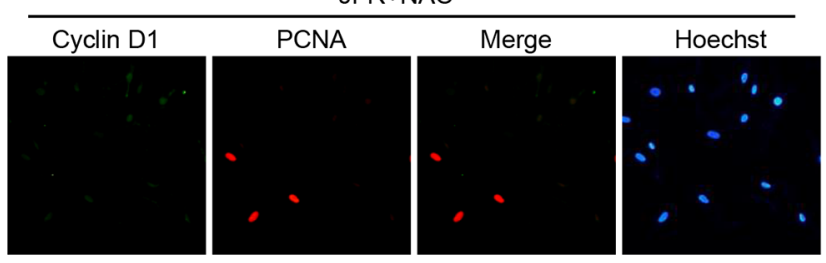

OFR+Mito-TEMPO
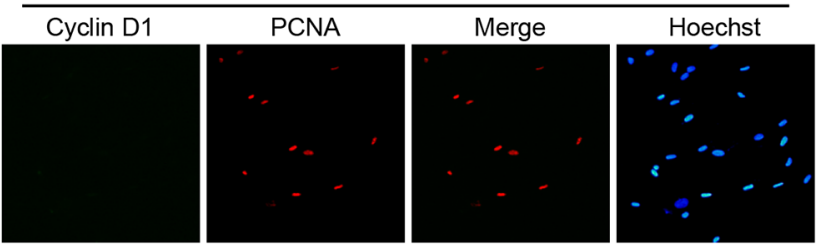

B

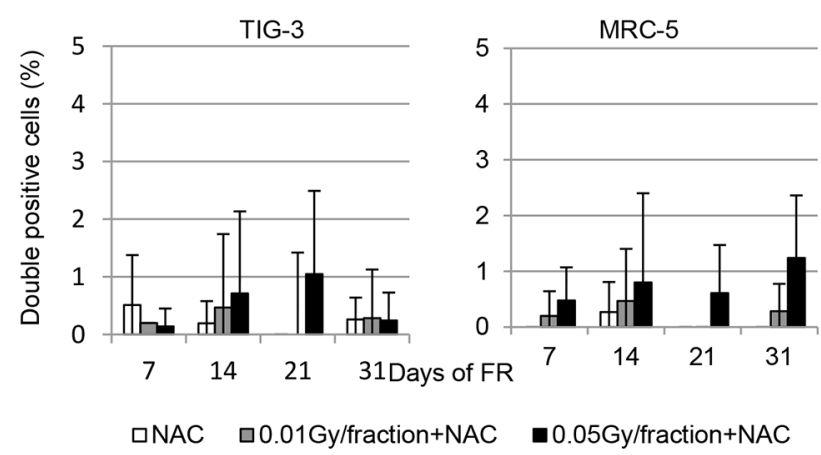

\section{Irradiation experiments}

Cells were irradiated using a $150-\mathrm{kVp}$ X-ray generator (Model MBR-1505R2, Hitachi, Tokyo, Japan) with a $0.5-\mathrm{mm} \mathrm{Cu}$ and $0.1-\mathrm{mm} \mathrm{Al}$ filter at a dose of 0.7 $\mathrm{Gy} / \mathrm{min}$. Low dose X-ray fractions ( 0.01 or $0.05 \mathrm{~Gy}$ ) were administrated twice a day and 5 days/week. Total doses delivered over 31 days were 0.46 Gy and 2.3 Gy for cells exposed to FR of 0.01 Gy and 0.05 Gy, respectively.

\section{ROS detection and mitochondrial mass measurement}

Cells were stained with $20 \mu \mathrm{M}$ DCFDA (Sigma) or 400nM MitoTracker Green FM (invitrogen) in minimum essential medium without serum for $30 \mathrm{~min}$ at $24 \mathrm{~h}$ after the last FR at indicated days. DCFDA-, MitoSOX-red- or
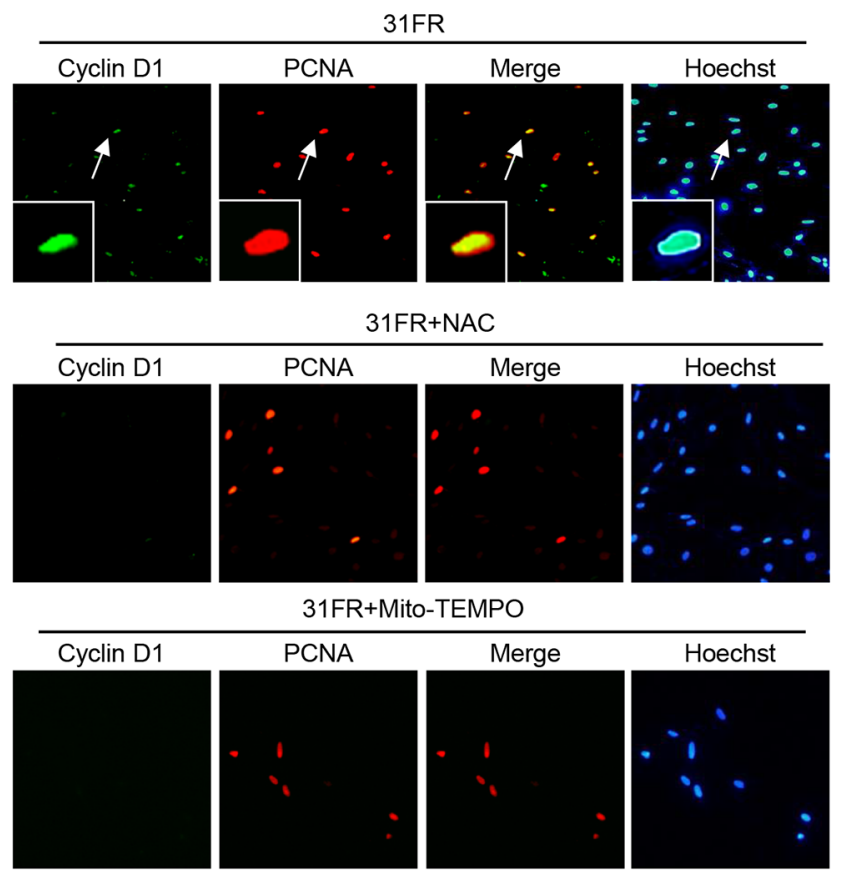

c

MRC-5

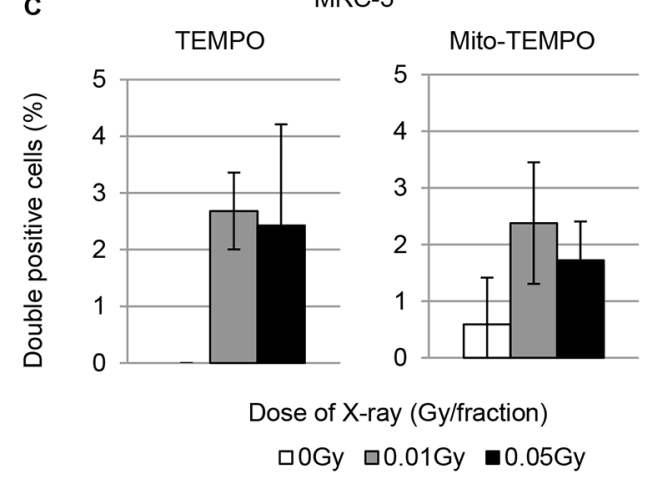

Figure 6. Inhibition of nuclear cyclin D1 accumulation by NAC in 31FR cells A. Immunofluorescence localization of cyclin D1 and PCNA is shown for control and 31FR MRC-5 cells with and without NAC treatment. DNA was stained with Hoechst. Magnified images are inserted. B. Cells double positive for cyclin D1 and PCNA were scored 24 hours following the indicated FR exposure. Data for MRC-5 and TIG-3 cells exposed to 0.01-Gy or 0.05-Gy fractions plus NAC are shown. 
MitoTracker Green FM-stained cells were quantified with a FACScan (Becton Dickinson, USA). Cells were placed on glass slides and cultured overnight. Cells on coverslips were stained with MitoSOX-red or MitoTracker Green FM according to the manufacturer's instructions (Invitrogen, Carlsbad, CA). Images were acquired using a CCD camera

\section{A MRC-5}

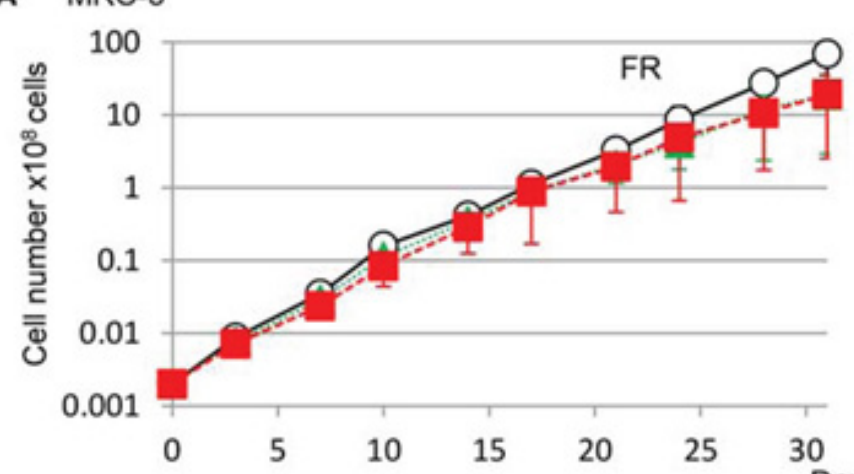

attached to a fluorescence microscope (Keyence, Osaka, Japan).

\section{Immunofluorescence}

Immunofluorescence stainings were performed as described [22, 32]. For PML immunostaining, cells were
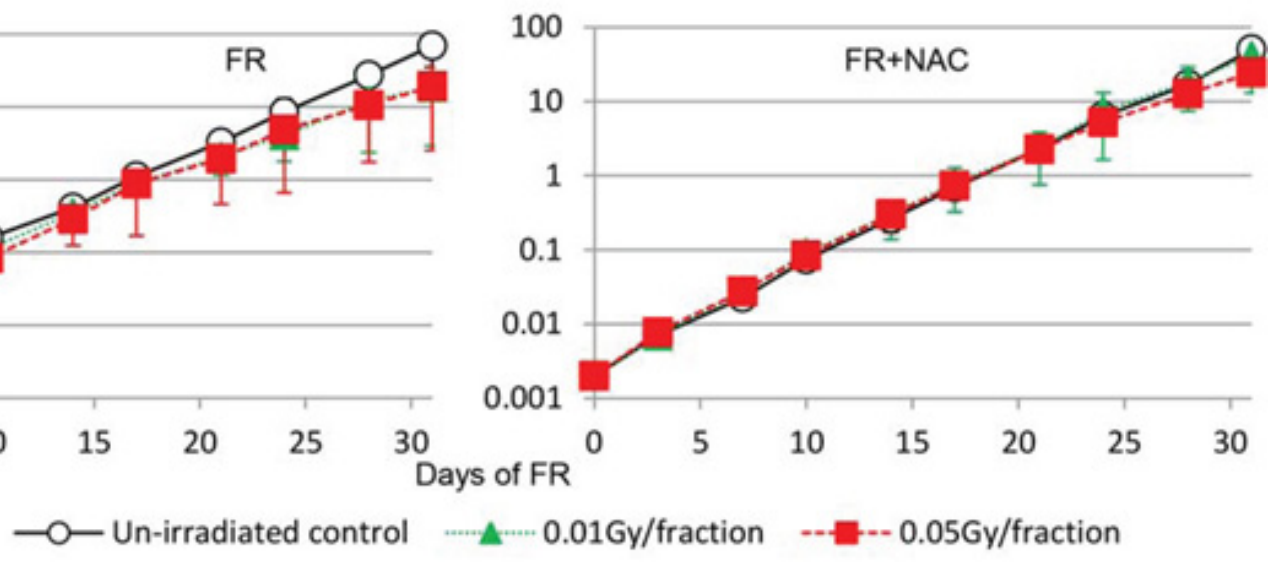

$0.01 \mathrm{~Gy} / \mathrm{fraction}$

-- - $-0.05 \mathrm{~Gy} /$ fraction

B
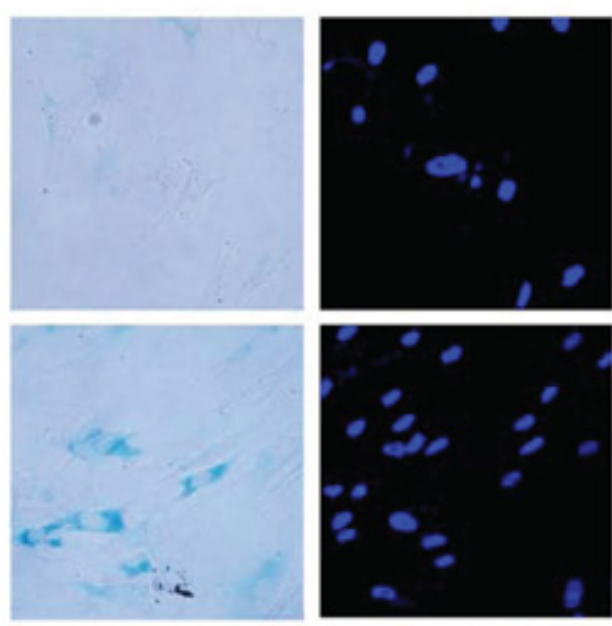

C Senescent cells
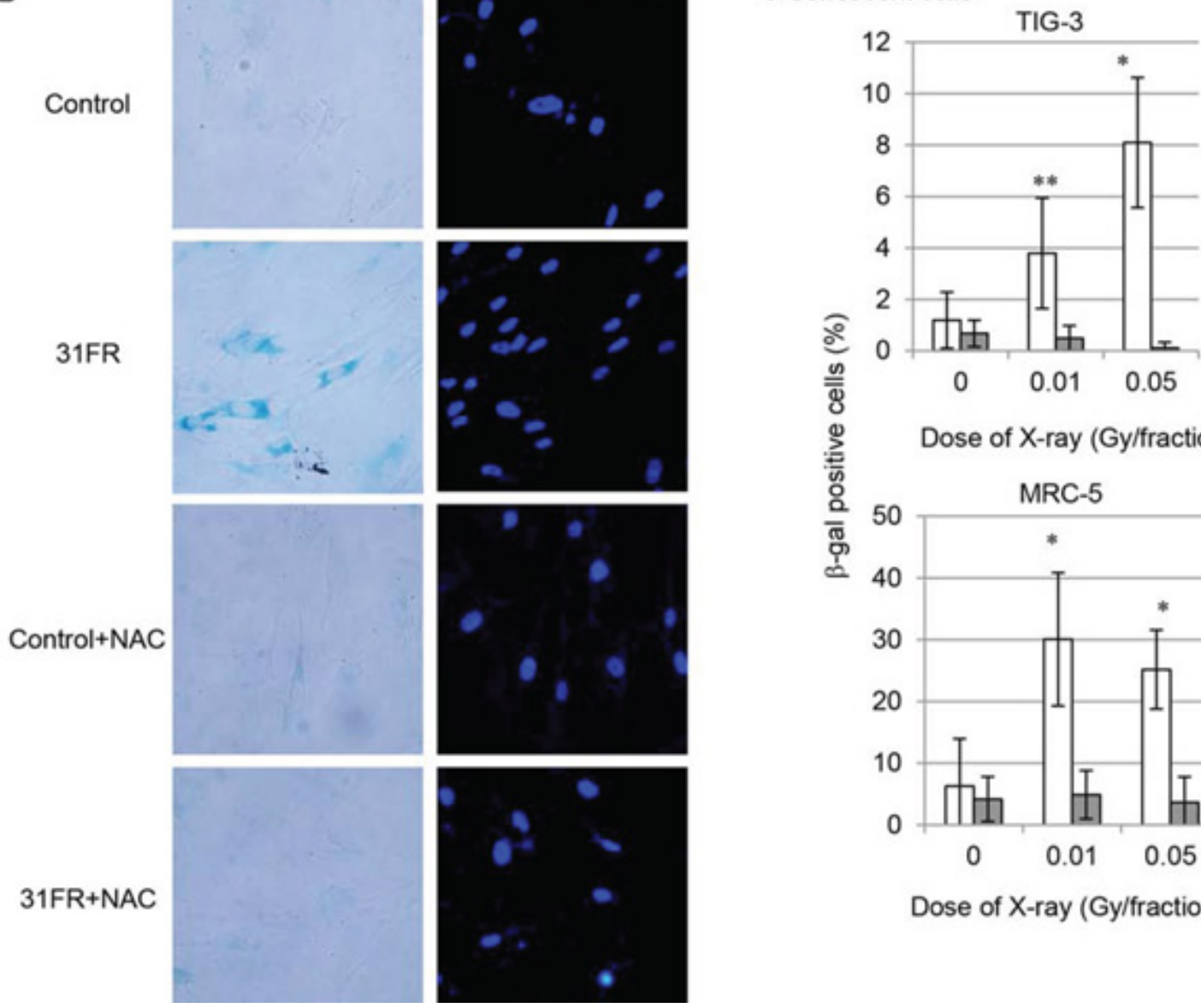
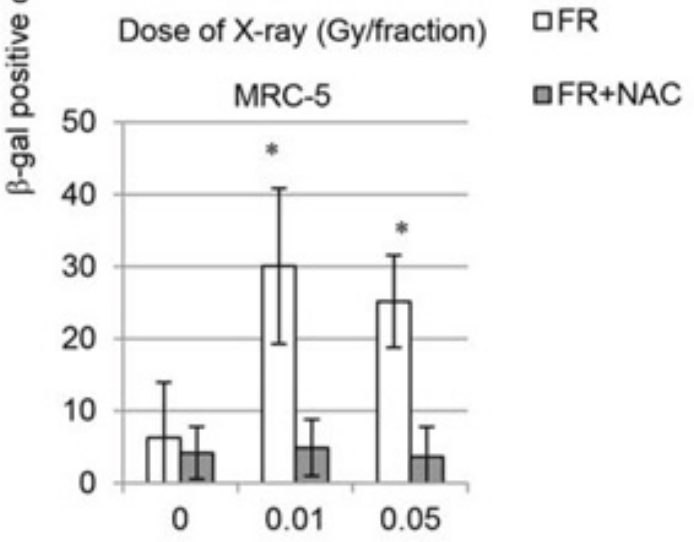

Dose of X-ray (Gy/fraction)

Figure 7. Growth retardation and cellular senescence after low-dose long-term FR A. Cell growth of unirradiated cells (open circles) and cells exposed to 0.01-Gy (open triangles) and 0.05-Gy (open squares) fractions. Growth curves for MRC-5 cells with and without NAC treatment are shown. B. Images of $\beta$-gal staining in unirradiated control and irradiated $31 \mathrm{FR}$ cells of MRC-5 with/without NAC treatment. C. Percentage of $\beta$-gal staining cells in unirradiated control and irradiated 31FR cells with/without NAC treatment. 
fixed with $4 \%$ formaldehyde for $10 \mathrm{~min}$ and permeabilized with $0.25 \%$ Triton X-100 for 5 min. Antibodies against PML (Milipore, MA), PCNA (Santa Cruz Biotechnology, Santa Cruz, CA) and cyclin D1 (Nichirei Bioscience, Tokyo, Japan) and secondary antibodies conjugated with Alexa Fluor 488 (Molecular Probes, Eugene, OR) or Cy-3 (Jackson ImmunoResearch Laboratories, West Grove, PA) were used. Cells were counterstained for DNA with Hoechst $33258(4 \mu \mathrm{g} / \mathrm{mL}$ in Vectashield mounting medium; Vector Laboratories, Burlingame, CA). Images were captured using a CCD camera attached to a fluorescence microscope (Keyence, Osaka, Japan). For each data point, $>50$ cells were counted from at least three independent samples.

\section{Western blot analyses}

Western blotting was performed as described [14]. Primary antibodies against $\beta$-actin (A2066, Sigma), AKT (Cell Signaling, Beverly, MA, USA), phospho-AKTSer473 (Cell Signaling), PP2AC (Cell Signaling) and a secondary goat anti-rabbit antibody conjugated with horseradish peroxidase (GE Healthcare, Little Chalfont, UK) were used. Protein bands were visualized using Chemi-Lumi One L Western blotting substrate (Nacalai Tesque), and band intensities were measured using Image Lab software (Bio-Rad).

\section{Measurement of GSH}

Total GSH was quantified using a Total Glutathione Quantification Kit (Dojindo) according to the manufacturer's protocol. OD values at $405 \mathrm{~nm}$ were measured with a microplate reader (Sunrise).

\section{Measurement of PP2A phosphatase activity}

PP2A phosphatase activity was measured from cell lysates $(100 \mu \mathrm{g})$ using a PP2A immunoprecipitation phosphatase assay kit according to the manufacturer's instructions (Millipore, Temecula, CA). DTT (1mM) was added to the lysis buffer for DTT-treated samples.

\section{Analysis of oxidative DNA damage in nuclear and mitochondria DNA}

Mitochondrial DNA and nuclear DNA were isolated by using a mitochondrial DNA isolation kit (Biovision) and a DNA Extraction WB kit (Wako Pure Chemical Industries, Osaka Japan), respectively. The isolated DNA was labeled with N-Amino-oxymethylcarbonyl-hydrazinoD-biotin by the manufacturer's instructions (Nucleostain DNA damage Quantification Kit; Dojindo). OD values at $650 \mathrm{~nm}$ were measured with a microplate reader (Sunrise). For 8-OHdG Assay, the isolated DNA was digested with nuclease P1 followed by the manufacturer's instructions (8-OHdG Assay Preparation reagent set; Wako Pure
Chemical Industries). The amounts of 8-OHdG were quantified using high performance liquid chromatographyelectrochemical detector (HPLC-ECD) as described previously [33].

\section{Diagonal electrophoresis}

Diagonal electrophoresis was carried out as followed by Moreno et.al. [34]. First dimension is performed under nonreducing conditions and second dimension under reducing conditions. Proteins with intermolecular disulfide bridges appear below the diagonal, while proteins with intramolecular disulfide bridges appear above the diagonal.

\section{Cell growth assay}

Cells $\left(2 \times 10^{5}\right)$ were seeded into $25-\mathrm{cm}^{2}$ flasks (Thermo Fisher Scientific, Waltham, MA), incubated overnight, and irradiated daily. Growth rates were monitored by counting cell numbers twice a week. When the total cell number exceeded $2 \times 10^{5}$, cells were subcultured to $2 \times 10^{5}$ cells in a new flask.

\section{Senescence}

Cellular senescence was examined by using the Senescence Detection Kit (BioVision) according to the manufacturer's instructions. Images were captured using a CCD camera attached to a fluorescence microscope (Keyence, Osaka, Japan). For each data point $>50$ cells were counted from at least three independent samples.

\section{Statistical analysis}

Error bars represent standard deviations. All experiments were repeated at least three times using independent samples. Student's $t$-test was used for statistical analyses. Single and double asterisks indicate significant differences at $p<0.01$ and $p<0.05$, respectively.

\section{ACKNOWLEDGMENTS}

The authors thank the staff of the National Institute of Public Health for assistance with the research.

\section{CONFLICTS OF INTEREST}

The authors declare no conflict of interest.

\section{GRANT SUPPORT}

This research was supported by a grant from the Japanese Ministry of Education and Science Houga (15K12220) and in part by NIFS Collaborative Research 
Program (NIFS13KOBA028). This work was performed at the Joint Usage/Research Center (RIRBM), Hiroshima University.

\section{REFERENCES}

1. Finkel T. From sulfenylation to sulfhydration: what a thiolate needs to tolerate. Science signaling. 2012; 5:pe10.

2. Liu X, Kim CN, Yang J, Jemmerson R and Wang X. Induction of apoptotic program in cell-free extracts: requirement for dATP and cytochrome c. Cell. 1996; 86:147-157.

3. Sena LA and Chandel NS. Physiological roles of mitochondrial reactive oxygen species. Molecular cell. 2012; 48:158-167.

4. Chandel NS. Mitochondria as signaling organelles. BMC biology. 2014; 12:34.

5. Mandal S, Lindgren AG, Srivastava AS, Clark AT and Banerjee U. Mitochondrial function controls proliferation and early differentiation potential of embryonic stem cells. Stem cells. 2011; 29:486-495.

6. Kim GJ, Chandrasekaran K and Morgan WF. Mitochondrial dysfunction, persistently elevated levels of reactive oxygen species and radiation-induced genomic instability: a review. Mutagenesis. 2006; 21:361-367.

7. Kim GJ, Fiskum GM and Morgan WF. A role for mitochondrial dysfunction in perpetuating radiation-induced genomic instability. Cancer research. 2006; 66:10377-10383.

8. Fridovich I. Superoxide radical and superoxide dismutases. Annual review of biochemistry. 1995; 64:97-112.

9. Meister A. Glutathione, ascorbate, and cellular protection. Cancer research. 1994; 54:1969s-1975s.

10. Nicholson KM and Anderson NG. The protein kinase B/Akt signalling pathway in human malignancy. Cellular signalling. 2002; 14:381-395.

11. Vivanco I and Sawyers CL. The phosphatidylinositol 3 -Kinase AKT pathway in human cancer. Nature reviews Cancer. 2002; 2:489-501.

12. Manning BD and Cantley LC. AKT/PKB signaling: navigating downstream. Cell. 2007; 129:1261-1274.

13. Zhou BB and Elledge SJ. The DNA damage response: putting checkpoints in perspective. Nature. 2000; 408:433-439.

14. Shimura T, Kakuda S, Ochiai Y, Nakagawa H, Kuwahara Y, Takai Y, Kobayashi J, Komatsu K and Fukumoto M. Acquired radioresistance of human tumor cells by DNA-PK/AKT/GSK3beta-mediated cyclin D1 overexpression. Oncogene. 2010; 29:4826-4837.

15. Gao T, Brognard $\mathrm{J}$ and Newton AC. The phosphatase PHLPP controls the cellular levels of protein kinase $\mathrm{C}$. The Journal of biological chemistry. 2008; 283:6300-6311.

16. Gao T, Furnari F and Newton AC. PHLPP: a phosphatase that directly dephosphorylates Akt, promotes apoptosis, and suppresses tumor growth. Molecular cell. 2005; 18:13-24.
17. Georgescu MM. PTEN Tumor Suppressor Network in PI3K-Akt Pathway Control. Genes \& cancer. 2010; 1:1170-1177.

18. Liu W, Akhand AA, Takeda K, Kawamoto Y, Itoigawa M, Kato M, Suzuki H, Ishikawa N and Nakashima I. Protein phosphatase 2A-linked and -unlinked caspase-dependent pathways for downregulation of Akt kinase triggered by 4-hydroxynonenal. Cell death and differentiation. 2003; 10:772-781.

19. Clerkin JS, Naughton R, Quiney C and Cotter TG. Mechanisms of ROS modulated cell survival during carcinogenesis. Cancer letters. 2008; 266:30-36.

20. Foley TD, Petro LA, Stredny CM and Coppa TM. Oxidative inhibition of protein phosphatase $2 \mathrm{~A}$ activity: role of catalytic subunit disulfides. Neurochemical research. 2007; 32:1957-1964.

21. Finkel T. Signal transduction by reactive oxygen species. The Journal of cell biology. 2011; 194:7-15.

22. Shimura T, Hamada N, Sasatani M, Kamiya K and Kunugita N. Nuclear accumulation of cyclin D1 following long-term fractionated exposures to low-dose ionizing radiation in normal human diploid cells. Cell cycle. 2014; 13:1248-1255.

23. Wu G, Fang YZ, Yang S, Lupton JR and Turner ND. Glutathione metabolism and its implications for health. The Journal of nutrition. 2004; 134:489-492.

24. Staal FJ, Roederer M, Herzenberg LA and Herzenberg LA. Intracellular thiols regulate activation of nuclear factor kappa B and transcription of human immunodeficiency virus. Proceedings of the National Academy of Sciences of the United States of America. 1990; 87:9943-9947.

25. Lee DH and Jacobs DR, Jr. Hormesis and public health: can glutathione depletion and mitochondrial dysfunction due to very low-dose chronic exposure to persistent organic pollutants be mitigated? Journal of epidemiology and community health. 2014.

26. Slezak BP, Hatch GE, DeVito MJ, Diliberto JJ, Slade R, Crissman K, Hassoun E and Birnbaum LS. Oxidative stress in female $\mathrm{B} 6 \mathrm{C} 3 \mathrm{~F} 1$ mice following acute and subchronic exposure to 2,3,7,8-tetrachlorodibenzo-p-dioxin (TCDD). Toxicological sciences. 2000; 54:390-398.

27. Gillett C, Fantl V, Smith R, Fisher C, Bartek J, Dickson C, Barnes D and Peters G. Amplification and overexpression of cyclin D1 in breast cancer detected by immunohistochemical staining. Cancer research. 1994; 54:1812-1817.

28. Russell A, Thompson MA, Hendley J, Trute L, Armes J and Germain D. Cyclin D1 and D3 associate with the SCF complex and are coordinately elevated in breast cancer. Oncogene. 1999; 18:1983-1991.

29. Shimura T, Ochiai Y, Noma N, Oikawa T, Sano Y and Fukumoto M. Cyclin D1 overexpression perturbs DNA replication and induces replication-associated DNA 
double-strand breaks in acquired radioresistant cells. Cell cycle. $2013 ; 12: 773-782$.

30. Dulic V, Drullinger LF, Lees E, Reed SI and Stein GH. Altered regulation of G1 cyclins in senescent human diploid fibroblasts: accumulation of inactive cyclin E-Cdk2 and cyclin D1-Cdk2 complexes. Proceedings of the National Academy of Sciences of the United States of America. 1993; 90:11034-11038.

31. Leontieva OV, Lenzo F, Demidenko ZN and Blagosklonny MV. Hyper-mitogenic drive coexists with mitotic incompetence in senescent cells. Cell cycle. 2012; 11:4642-4649.

32. Shimura T, Toyoshima M, Adiga SK, Kunoh T, Nagai H, Shimizu N, Inoue M and Niwa O. Suppression of replication fork progression in low-dose-specific p53-dependent S-phase DNA damage checkpoint. Oncogene. 2006; 25:5921-5932.

33. Inaba Y, Koide S, Yokoyama $\mathrm{K}$ and Karube I. Development of urinary 8-hydroxy-2'-deoxyguanosine (8-OHdG) measurement method combined with SPE. Journal of chromatographic science. 2011; 49:303-309.

34. Moreno ML, Escobar J, Izquierdo-Alvarez A, Gil A, Perez S, Pereda J, Zapico I, Vento M, Sabater L, Marina A, Martinez-Ruiz A and Sastre J. Disulfide stress: a novel type of oxidative stress in acute pancreatitis. Free radical biology \& medicine. 2014; 70:265-277. 